\title{
Um caso de dermatose perfurante adquirida
}

Ana Dantas*, Gonçalo Melo**

\section{RESUMO}

Apresenta-se um caso de dermatose perfurante adquirida, frequentemente associada com diabetes mellitus e insuficiência renal crónica. Trata-se de uma senhora de 61 anos, diabética, com um quadro de pápulas e nódulos umbilicados, com centro queratósico e muito pruriginosos, localizados na face extensora dos membros inferiores. O diagnóstico de dermatose perfurante adquirida e a respectiva terapêutica foram concretizados apenas 6 anos após a apresentação do quadro. Este artigo visa alertar para a existência de uma patologia pouco diagnosticada, com impacto psico-social considerável e com perspectiva de se tornar cada vez mais prevalente.

Palavras-chave (Termos MeSH): Collagen Diseases; Skin Diseases; Diabetes Mellitus.

A s doenças perfurantes são patologias em que ocorre migração transepidérmica de componentes da matriz extracelular da derme, por inflamação e/ou degeneração. Existem quatro tipos clássicos: Doença de Kyrle, Elastose perfurante serpiginosa, Colagenose reactiva perfurante (CRP) e Foliculite perfurante. As formas adquiridas são consideradas uma entidade distinta, a dermatose perfurante adquirida (DPA). ${ }^{1} \mathrm{~A}$ sua prevalência atinge os $10 \%$ nos hemodialisados do Reino Unido e da América do Norte. ${ }^{1-3}$ Na população diabética estima-se em $50 \%$. No entanto, a DPA é pouco conhecida e pouco diagnosticada. ${ }^{2}$ Os principais factores associados são a diabetes mellitus tipo 2 (DM2), a insuficiência renal crónica (IRC), sobretudo se em hemodiálise (HD), a hipertensão arterial crónica e a nefropatia a Imunoglobulina A. ${ }^{2-8}$ É mais comum na DM2 (72,7\%) do que na DM tipo $1(27,3 \%){ }^{1,3}$ Surge em média 20,3 anos após o início da DM sem necessidade de HD, 14,5 anos após o início da DM com necessidade de HD, e 5,3 anos após o início da HD. ${ }^{1}$ Dois estudos mostraram que a grande maioria dos diabéticos $(90,9 \%)$ tem pelo menos uma complicação quando surge a CRP: nefropatia (90,9\%), retinopatia $(81,8 \%)$, doença arterial periférica $(45,5 \%)$

*Interna do Internato de Medicina Geral e Familiar. Unidade de Saúde Familiar Tílias, Centro de Saúde de Sete Rios, ACES Lisboa Norte

**Assistente de Medicina Geral e Familiar. Unidade de Saúde Familiar Tílias, Centro de Saúde de Sete Rios, ACES Lisboa Norte ou neuropatia $(45,5 \%) .{ }^{1,3}$ Nos indivíduos susceptíveis, o trauma superficial (frequentemente a coceira devido ao prurido) provoca necrose das fibras de colagénio da derme com consequente eliminação transepidérmica. Trata-se de um processo auto-limitado, mas recorrente. ${ }^{2} \mathrm{~A}$ idade média de apresentação desta DPA é os 56 $\operatorname{anos.~}^{3}$ As lesões típicas são placas, pápulas e nódulos com 4 a $10 \mathrm{~mm}$, que umbilicam em 3 a 5 semanas e regridem em 6 a 8 semanas, ficando com um tampão queratósico muito aderente cujo destacamento pode sangrar. Posteriormente resta apenas uma cicatriz ou mancha hiperpigmentada. ${ }^{1-5}$ As lesões localizam-se predominantemente nas superfícies extensoras dos membros e das mãos $(63,6 \%)$, tronco $(59,1 \%)$ e cabeça $(27,3 \%)$, sendo mais frequente a localização múltipla $(72,7 \%)$. Os sintomas mais frequentemente associados são o prurido $(72,7 \%)$ e a dor $(9,1 \%) .{ }^{4}$ As lesões são reproduzíveis em $31 \%$ dos casos (fenómeno de Koebner).$^{1-4} \mathrm{O}$ diagnóstico é clínico e histopatológico, implicando o cumprimento dos critérios de Faver: histopatologia com eliminação transepidérmica de fibras de colagénio necróticas basofílicas numa depressão epidérmica em forma de chávena; pápulas ou nódulos umbilicados com centro queratósico aderente; e início depois dos 18 anos. ${ }^{1} \mathrm{O}$ diagnóstico diferencial da CRP inclui as outras doenças perfurantes, o prurigo nodular e o líquen plano hipertrófico. ${ }^{2,3}$ As terapêuticas disponíveis são: emolientes; ; ${ }^{1,5}$ queratolíticos; ${ }^{2,8,9}$ anti-histamí- 
nicos; ${ }^{2,5,8}$ antibióticos tópicos e per os; ${ }^{2}$ corticosteróides tópicos; $;^{1,8,9}$ retinóides tópicos e per os; $;^{2,5,8,9}$ alopurinol; ${ }^{2,5,8,9}$ talidomida; ${ }^{2}$ psoraleno e radiação ultravioleta do tipo A e B; ${ }^{1,2,5,8,9}$ crioterapia; ${ }^{1,8,9}$ e estimulação eléctrica dos nervos. ${ }^{8} \mathrm{~A}$ única possível complicação é a impetiginação das lesões. ${ }^{1}$ Há casos de remissão espontânea, após o controlo da DM, após transplante renal e após interrupção da HD. No entanto, a recorrência é a regra. ${ }^{1,2,9}$

M é uma senhora de 61 anos, caucasiana, casada, habilitada com o $4 .^{\circ}$ ano de escolaridade, empregada doméstica, natural de Portalegre e residente em Lisboa. Encontra-se na fase VI do ciclo de Duvall e no $4 .^{\circ}$ nível da classificação de Graffar. Os seus problemas de saúde relevantes incluem DM tipo 2 desde os 39 anos (sem qualquer complicação) e hipertensão arterial controlada desde os 43 anos. Aos 54 anos, M iniciou um quadro de pápulas e nódulos, com 6 a $10 \mathrm{~mm}$, umbilicados, com centro queratósico, muito pruriginosos, na face extensora da perna direita, que cicatrizavam espontaneamente após algumas semanas com hiperpigmentação residual. Este quadro recorria frequentemente, tendo sido medicada com beclometasona tópica mas sem melhoria. Quatro anos depois, por extensão do quadro à outra perna, foi enviada à consulta externa de Dermatologia do seu hospital de referência. Dois anos mais tarde, e apesar do seguimento regular na Dermatologia e da terapêutica com corticosteróide tópico, o quadro aumentou de intensidade, levando $\mathrm{M}$ a recorrer novamente ao seu médico de família (ver Figura $1 \mathrm{e}$ 2). M encontrava-se preocupada com a natureza das lesões e sobretudo com o impacto social das mesmas, recusando-se a usar peças de vestuário que expusessem as pernas. Foram colocadas as hipóteses diagnósticas de prurigo nodular e doença perfurante, tendo sido prescritos calamina e eritromicina tópicos bem como hidroxizina oral. Concomitantemente, foi solicitada uma biopsia cutânea noutro centro hospitalar. Quatro meses depois, registava-se completa regressão do quadro (ver Figura 3) e a biopsia confirmava o diagnóstico de DPA. Desde então, M sofreu apenas uma recorrência que cedeu em poucos dias à terapêutica anteriormente prescrita.

O quadro clínico de M era típico de DPA. No entanto, é raro as lesões cutâneas surgirem sem a presença de qualquer complicação da DM. Este facto, associado

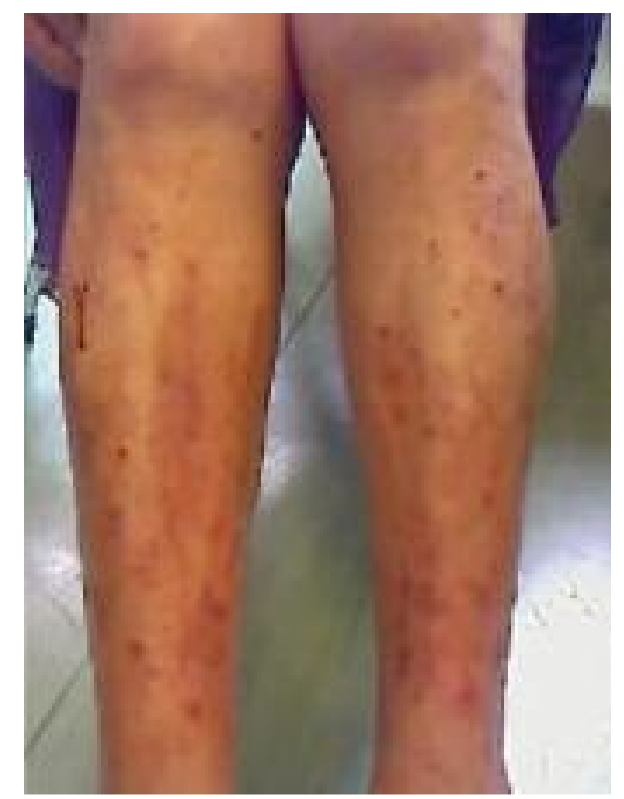

Figura 1. Membros inferiores de $M$ com lesões cutâneas.

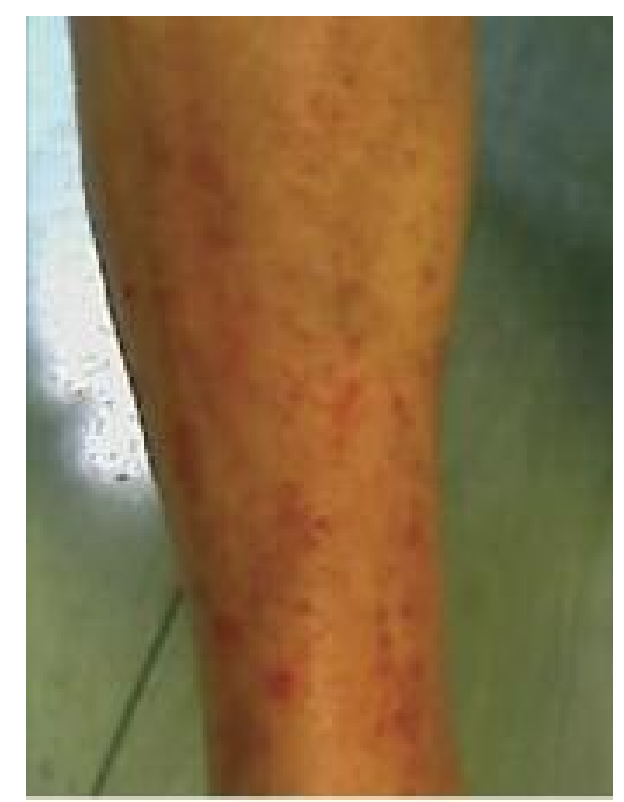

Figura 2. Face anterior do membro inferior direito de $M$ com lesões cutâneas

à pouca divulgação desta patologia entre os profissionais de saúde, condicionou um importante atraso no diagnóstico e na implementação de terapêutica adequada, com prejuízo para M. Esperamos que este artigo sirva de alerta para a existência de uma patologia 


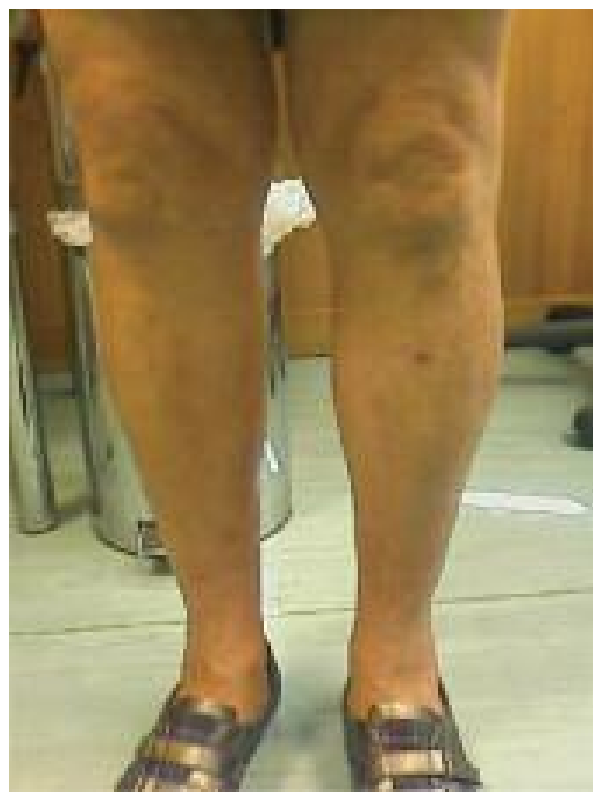

Figura 3. Membros inferiores de $M$ após a resolução das lesões cutâneas

pouco diagnosticada, com impacto psico-social considerável e com perspectiva de se tornar cada vez mais prevalente devido ao aumento da prevalência dos factores a si associados (DM, IRC, HD e hipertensão arterial).

\section{REFERÊNCIAS BIBLIOGRÁFICAS}

1. Kawakami T, Saito R. Acquired reactive perforating collagenosis asso- ciated with diabetes mellitus: eight cases that meet Faver's criteria. Br J Dermatol 1999 Mar; 140 (3): 521-4.

2. Arias M, La Forgia M, Buonsante ME, Portaluppi M, Kien MC, Pellerano G. Dermatosis perforante adquirida en pacientes con insuficiencia renal crónica. Comunicación de tres casos y revisión de la literatura. Dermatología Argentina 2007; 13(3): 184-9.

3. Saray $Y$, Seçkin D, Bilezikçi B. Acquired perforating dermatosis: clinicopathological features in twenty-two cases. J Eur Acad Dermatol Venereol 2006 Jul; 20 (6): 679-88.

4. Velázquez GE, Marín HE, Vidal FA. Colagenosis perforante reactiva: comunicación de un caso. Rev Cent Dermatol Pascua 2004 Sep-Dic; 13 (3): 154-7.

5. Cooper S, Asher RG. Reactive perforating collagenosis [Internet]. Emedicine 2009 Disponível em: http://emedicine.medscape.com/article/1074803-overview [acedido em 31/12/2009].

6. Yadav MK, Sangal BC, Bhargav P, Jai PR, Goyal M. Reactive perforating collagenosis. Indian J Pathol Microbiol 2009 Jan-Mar; 52 (1): 106-7.

7. Sabater Marco V, Salgado Jaaldo E. Colagenosis perforante reactiva adquirida - estudio de un caso de origen traumático. Rev Esp Patol 2007; 40 (1): 53-6.

8. Schmults $C A$. Acquired reactive perforating collagenosis. Dermatol Online J 2002 Oct; 8(2): 8.

9. Yancovitz M, Johnson H, Wang N, Pomeranz MK. Perforating collagenosis. Dermatol Online J 2008 Oct 15; 14 (10): 14.

\section{CONFLITOS DE INTERESSE}

Os autores declaram não possuir qualquer tipo de conflito de interesses.

\section{ENDEREÇO PARA CORRESPONDÊNCIA}

Rua Padre Carlos dos Santos, Bloco B, 1., 1500-901 Lisboa

E-mail: dantas80@gmail.com

Recebido em 16/11/2010

Aceite para publicação em 16/06/2011

\section{ABSTRACT}

\section{A CASE OF ACQUIRED PERFORATING DERMATOSIS}

We present a case of acquired perforating dermatosis (also known as acquired perforating collagenosis) which is frequently associated with diabetes mellitus and chronic renal failure. A 61 year old diabetic woman had a history of umbilicated, pruritic papules and nodules, with a central keratotic plug, located on the extensor surface of the lower limbs. The diagnosis of acquired perforating dermatosis was made six years after the initial clinical presentation. This article seeks to alert physicians to the existence of this under-diagnosed condition with considerable psychosocial impact.

Keywords (Termos MeSH): Collagen Diseases; Skin Diseases; Diabetes Mellitus. 